\title{
Promoter methylation, mRNA expression of goat tumor-associated genes and mRNA expression of DNA methyltransferase in enzootic nasal tumors
}

\author{
ZIFANG QUAN $^{1}$, NI YE ${ }^{1}$, ZHONGXIANG HAO ${ }^{1}$, CAIFANG WEN ${ }^{1}$, HONG LIAO ${ }^{1}$, MANLI ZHANG ${ }^{1}$,

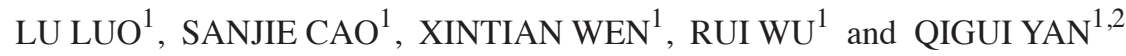 \\ ${ }^{1}$ College of Veterinary Medicine; ${ }^{2}$ Key Laboratory of Animal Disease and Human Health of Sichuan Province, \\ Sichuan Agricultural University, Chengdu, Sichuan 611130, P.R. China
}

Received May 13, 2014; Accepted January 29, 2015

DOI: $10.3892 / \mathrm{mmr} .2015 .4184$

\begin{abstract}
The aim of the present study was to investigate the promoter methylation status and mRNA expression of goat tumor-associated genes, in addition to the mRNA expression of DNA methyltransferase genes in enzootic nasal tumors (ENT). Methylation-specific polymerase chain reaction and SYBR Green reverse transcription-quantitative polymerase chain reaction were used to detect the methylation status and the mRNA expression levels of DNA methyltransferases (DNMTs), $\mathrm{O}^{6}$-methylguanine-DNA methyltransferase (MGMT), the tumor suppressor genes P73, P53, GADD45G, CHFR and THBS1, the transcription factor CEBPA, the proto-oncogenes KRAS, NRAS and C-myc and EGFR in 24 nasal tumor tissue samples and 20 normal nasal epithelia tissue samples. The associations between promoter methylation and DNMT, and promoter methylation and mRNA expression of the genes were analyzed. The results indicated that the expression levels of DNMT1 increased by $56 \%$ compared with those in normal nasal epithelial tissues, while MGMT, DNMT3a and DNMT3b had similar expression levels in the two tissue types. The expression levels of P53 decreased by $36.8 \%$ and those of THBS1 by $43 \%$, while C-myc increased by 2.9-fold and CEBPA by 2-fold compared with that in normal nasal epithelial tissues. GADD45G, P73, CHFR and NRAS were observed to have similar expression levels in the two tissue types. However, no expression was observed for EGFR and KRAS. CHFR, GADD45G and THBS1 were identified to be methylated in tumor suppressor genes. The methylation expression rate of the CHFR gene was $\sim 60 \%$ in the two tissue types and for THBS1 it was $100 \%$ in the nasal tumor tissues
\end{abstract}

Correspondence to: Professor Qigui Yan, College of Veterinary Medicine, Sichuan Agriculture University, 211 Huimin Road, Chengdu, Sichuan 611130, P.R. China

E-mail: yanqigui@126.com

Key words: goat, enzootic nasal tumor, DNA methyltransferase, promoter methylation, mRNA expression as opposed to $20 \%$ in the normal nasal epithelial tissues. The exhaustive methylation expression rate of GADD45G was $62.5 \%$ and the partial methylation expression rate was $37.5 \%$ in nasal tumor tissue, while no methylation was observed in normal nasal epithelial tissues. C-myc was the only gene identified to be methylated amongst proto-oncogenes. The methylation expression rate of $\mathrm{C}$-myc was $87.5 \%$ in nasal tumor tissues and $15 \%$ in normal nasal epithelial tissues. The methylation expression rate of CEBPA was $100 \%$ in nasal tumor tissues and $40 \%$ in normal nasal epithelial tissues. The methylation expression rate of the EGFR gene was $\sim 80 \%$ in the two tissues. In summary, the present study identified abnormal methylation of the C-myc, CEBPA, GADD45G and THBS1 genes in nasal tumor tissues. The expression levels of DNMT1, C-myc and CEBPA were upregulated and the expression of P53 and THBSI were downregulated in nasal tumor tissues, with a significant difference between the two groups $(\mathrm{P}<0.05)$. Therefore, it is suggested that these six genes may be used as diagnostic marker candidates for ENT. The results may serve as a foundation for screening of tumor-specific markers for early diagnosis of ENT and further investigate the epigenetic mechanisms of enzootic nasal tumor virus (ENTV)-induced nasal epithelium cell carcinoma.

\section{Introduction}

Enzootic nasal tumor (ENT) is caused by the enzootic nasal tumor virus (ENTV) and is a chronic, progressive, sexually transmitted disease. Excluding Australia and New Zealand, ENT has spread throughout the majority of countries which keep goats (1). At present, an effective method for the early diagnosis of ENT remains to be developed, and affected goats are only removed subsequent to the appearance of symptoms (1). Due to the fact that goats with ENT cannot be distinguished from those which are healthy, disease spreads in the group, causing a greater number of goats to become infected, which can threaten the security of the population. An increasing number of studies have demonstrated that abnormal DNA methylation is closely associated with the presence of neoplasms as well as autoimmune and neurodegenerative diseases (1-3). DNA methylation involves the addition of 
a methyl group to the carbon of the cytosine pyrimidine ring in 5-position via the action of DNA methyltransferase (DNMT) (2-4). Abnormal DNA methylation often leads to tumor formation, which is closely associated with the occurrence of cancer (5). Investigating the promoter methylation status and mRNA expression of goat tumor-associated genes and DNMT genes in primary tumors in ENT can provide a foundation for screening of tumor-specific markers for early diagnosis of ENT and further investigations into the epigenetic mechanisms of ENTV-induced nasal epithelial cell carcinoma.

\section{Materials and methods}

Tissue specimens. A total of 24 nasal tumor tissue (from goats with ENT) specimens and 20 normal nasal epithelial tissue specimens from healthy animals were obtained from Yiyou farms (Sichuan, China). The animals $\sim 1$ year old and were sacrificed by the owner prior to sample collection. The present study was approved by the National Institute of Animal Health Animal Care and Use Committee at Sichuan Agricultural University (Ya'an, China; approval no. 2010-020).

Quarantine of control groups. A pair of primers were designed and synthesized based on the reported genomic RNA sequence of ENTV and the genetic variation range of gag. Sequences of the polymerase chain reaction (PCR) primers were: gag $(850 \mathrm{bp})$ forward, 5'-TTCCTCGCCACT ACTCTTG-3' and reverse, 5'-AGTCGCTGTGCTTGTTTC A-3 $(6,7)$. According to the manufacturer's instructions, RNA was extracted, then reverse transcription and PCR amplification were conducted. Amplified PCR products were evaluated by $2 \%$ agarose gel [Tiangen Biotech (Beijing) Co., Ltd., Beijing, China] electrophoresis.

mRNA expression of DNMT. Total RNA from nasal tumor tissues and normal nasal epithelial tissues was isolated using the RNeasy Micro kit (Sigma-Aldrich Shanghai Trading Co., Ltd., Shanghai, China) and the quantities were determined spectrophotometrically (722n; Shanghai Jinghua Science \& Technology Instruments Co., Ltd., Shanghai, China). First-strand cDNA was synthesized from $2 \mu \mathrm{g}$ total RNA using the RevertAid kit (Thermo Fisher Scientific, Waltham, MA, USA). The PCR conditions included an initial cDNA synthesis reaction at $42^{\circ} \mathrm{C}$ for $1 \mathrm{~h}$ using the RevertAid kit, followed by traditional PCR amplification. The positive standard was prepared according to the conventional methods and the recombinant plasmids were sent to a sequencing facility (Shanghai Invitrogen Biotechnology Co., Ltd., Shanghai, China). Subsequent to sequencing, the results were analzyed using the Basic Local Alignment Search Tool algorithm (BLAST; http://blast.ncbi.nlm.nih.gov/Blast.cgi) and compared with the corresponding subtypes.

Optimal reaction conditions of reverse transcription-quantitative polymerase chain reaction (RT-qPCR) were used. The RT-qPCR was performed on a CFX96 Real-Time PCR Detection system (Bio-Rad Laboratories, Inc., Hercules, CA, USA). A total of $2 \mu 1$ positive standard, 12.5 $\mu 1 \mathrm{SYBR}^{\circledR}$ Premix Ex Taq ${ }^{\mathrm{TM}}$ II (Tli RNase H Plus; 2X) (Takara Biotechnology Co., Ltd., Dalian, China) , $1 \mu$ l each

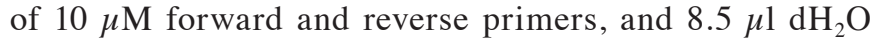
were added to each reaction well. The PCR conditions included a denaturation step for $30 \mathrm{sec}$ at $95^{\circ} \mathrm{C}$ and 40 cycles of $5 \mathrm{sec}$ at $95^{\circ} \mathrm{C}$ and $30 \mathrm{sec}$ at a temperature gradient from $55-64^{\circ} \mathrm{C}$. Following the last cycle, melting curve analysis was performed at $65-95^{\circ} \mathrm{C}$ with an increment of $0.5^{\circ} \mathrm{C} / \mathrm{sec}$. The housekeeping gene 18S-rRNA was used as an internal control. The sequences of the PCR primers were as follows: DNMT3a (191 bp) forward, 5'-ACGGAGAAGCCTAAG GTCAAG-3' and reverse, 5'-TGACGAAGGACCTTACGC G-3'; DNMT3b (212 bp) forward, 5'-CAGACAGCACCG AGTATCAGG-3' and reverse, 5'-CAACCACGTAACCCT AAC-3'; DNMT1 (176 bp) forward, 5'-CCCCAGGATTAC AAGGAAG-3' and reverse, 5'-CTGGATGTAACTCGACGT CTCT-3'; MGMT (101 bp) forward, 5'-CAACCCTATTCC CATCCTCATCC-3' and reverse, 5'-GCACTTCCTCACCGA CGACC-3'; 18S-rRNA (198 bp) forward, 5'-GAGAAACGG CTACCACATC-3' and reverse, 5'-GCTATTGGAGCTGGA ATTAC-3'. The optimal annealing temperature of each gene was determined using the $\mathrm{Ct}$ values, the highest fluorescence value and melting curve analysis.

The standard and melting curves were established using RT-qPCR amplification of the positive standard with a multiple proportion dilution $\left(1 \times 10^{1} \sim 1 \times 10^{6}\right.$ copies $\left./ \mu 1\right)$. RT-qPCR amplified the positive standard with an optimal annealing temperature. The experiments were repeated three times to evaluate the repeatability and reproducibility. The data were analyzed using the $2^{-\Delta \Delta \mathrm{Ct}}$ method (8) and SPSS, version 13.0 (SPSS, Inc., Chicago, IL, USA).

mRNA expression of goat tumor-associated genes. For detection of tumor-associated genes, the same methods were used as for detecting the expression of DNMT. The sequences of the PCR primers used were as follows: P73 (219 bp) forward, 5'-CCCTCCAACACCGACTACCC-3' and reverse, 5'-GGTCACATGCTCCGCCTTCTTAT-3'; P53 (148 bp) forward, 5'-CCACCATCCACTACA ACT TCA-3' and reverse, 5'-CCAGGACAGGCACAAACACG-3'; KRAS (198 bp) forward, 5'-GGCTCAGGACTTAGCAAG AA-3' and reverse, 5'-CGTCAACACCCAGATTACAT-3'; NRAS (118 bp) forward, 5'-TCAGTGAGCCAATTAGCA TC-3' and reverse, 5'-TAACCGACTTCTTTCCTTGC'; GADD45G (325 bp) forward, 5'-ATCTCACCACCTCTT GCTCG-3' and reverse, 5'-AGTCGTTGACGCTGCGG CTC-3'; EGFR (254 bp) forward, 5'-TGTGACTTGCGTTGA TAGAA-3' and reverse, 5'-CGGCGTTGCTGCGTGAAT-3'; CHFR (202 bp) forward, 5'-AACAAGAGCCATCAACCG-3' and reverse, 5'-AGGAGATGAGCACCGAAG-3'; C-myc (136 bp) forward, 5'-ACATCCTGTCGGTCCAAGCA-3' and reverse, 5'-CCTCCCTCCAATAGGTCAAT-3'; THBS1 (354 bp) forward, 5'-CTGTGCGGGCGGAGAAAGGT-3' and reverse, 5'-TGCTGAGTCTGGCGATGCTG-3'.

Assessment of the promoter methylation status of goat tumor-associated genes. Total DNA from nasal tumor tissues, normal nasal epithelial tissues and peripheral-blood cells was isolated using the Mammalian Genomic DNA Extraction kit (Takara Biotechnology Co., Ltd.) and the quantities were determined spectrophotometrically. The $\mathrm{CpG}$ island of peripheral-blood cell DNA was modified 


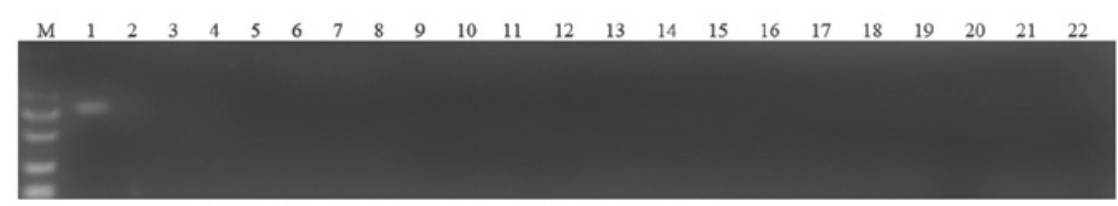

Figure 1. Electrophoretic analysis of samples from healthy animals by conventional polymerase chain reaction. 1, Positive control of enzootic nasal tumor virus; 2, negative control; 3-22, samples from healthy animals; M, DL2000 marker.

A

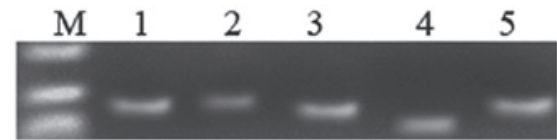

$\mathbf{B}$

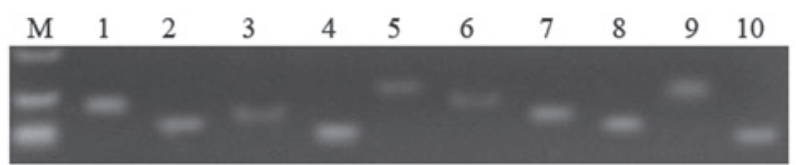

Figure 2. Electrophoretic analysis of recombinant plasmids amplified by conventional polymerase chain reaction in cancerous tissue samples. (A) Lane 1, DNMT3a; 2, DNMT3b; 3, DNMT1; 4, MGMT; 5, 18S-Rrna; 6, negative control; M, DL2000 marker. (B) Lane 1, P73; 2, P53; 3, KRAS; 4, NRAS 5, GADD45G; 6, EGFR; 7, CHFR; 8, C-myc; 9, THBS1; 10, CEBPA; M, DL2000 marker.

using $\mathrm{CpG}$ methyltransferase (Zymo Research, Irvine, CA, USA) and used as the positive control template of methylation following modification. The DNA of nasal tumor tissues, normal nasal epithelial tissues, peripheral blood cells and the DNA modified by $\mathrm{CpG}$ methyltransferase was modified using the EZ DNA Methylation-Gold ${ }^{\mathrm{TM}}$ Kit (Zymo Research). The DNA of peripheral blood cells was used as unmethylated positive-control template following modification.

A total of $2 \mu \mathrm{l}$ DNA sample, $10 \mu \mathrm{l}$ EmeraldAmp PCR Master Mix (2xPremix) (Takara Biotechnology Co., Ltd.), $0.5 \mu \mathrm{l}$ each of $20 \mu \mathrm{M}$ forward and reverse primers and $7 \mu \mathrm{l} \mathrm{dH}_{2} \mathrm{O}$ was added to each reaction well. Amplifications were performed using the following methylation-specific PCR (MSP) conditions: $95^{\circ} \mathrm{C}$ for $5 \mathrm{~min}, 35$ cycles of $95^{\circ} \mathrm{C}$ for $30 \mathrm{sec}, 57^{\circ} \mathrm{C}$ (methylation, $\mathrm{M}$ ) or $55^{\circ} \mathrm{C}$ (unmethylation, $\mathrm{U})$ for $45 \mathrm{sec}$, and $30 \mathrm{sec}$ at $72^{\circ} \mathrm{C}$. Following the last cycle, a final extension was performed at $72^{\circ} \mathrm{C}$ for $10 \mathrm{~min}$. The sequences of the MSP primers were as follows: GADD45G (M) (134 bp) forward, 5'-CGGGGTTTT TTTATTTTA TTTAGC-3' and reverse, 5'-GACTCGTTTACATTTCTA TACCGAA-3'; GADD45G (U) (134 bp) forward, 5'-TGG, GGTTTTTTTATTTTATTTAGTG-3' and reverse, 5'-AAC TCATTTACATTTCTATACCAAA-3'; P73 (M) (274 bp) forward, 5'-TAGTGAATTTTATTTTTTAGTTCGT-3' and reverse, 5'-AACCTA AAACTCTAAATTACTCCGC-3'; P73 (U) (273 bp) forward, 5'-TAGTGAATTTTATTTTTT AGTTTGT-3' and reverse, 5'-ACCTAAAACTCTAAATTA CTCCACC-3'; CEBPA (M) (165 bp) forward, 5'-ATGGGT TTGTTTTTTGTTTTTTTC-3' and reverse, 5'-ACGTAA TTACTACCTCTCCCAAC G-3'; CEBPA (U) (166 bp) forward, 5'-ATGGGTTTGTTTTTTGTTTTTTTT-3' and reverse, 5'-CACATA ATTACTACCTCTCCCAACAA-3'; KRAS (M) (184 bp) forward, 5'-ATTTTTGTTAATTGT TTTGGAGAAC-3' and reverse, 5'-TACTCTATATCCTAT AAACCCACCG-3'; KRAS (U) (180 bp) forward, 5'-TTT
TGTTAATTGTTTTGGAGAATGA-3' and reverse, 5'-CTC TATATCCTATAAACCCACCACA-3'; NRAS (M) (149 bp) forward, 5'-TTTTTTTTATTTTTTCGTATTCGA-3' and reverse, 5'-TCTACTCCTCTTAAA ACATTACGCT-3'; NRAS (U) (150 bp) forward, 5'-TTTTTTTATTTTTTTGTA TTTGA-3' and reverse, 5'-TTCTACTCCTCTTAAAACATT ACACT-3'; P53 (M) (198 bp) forward, 5'-TGTATAGTATAT AATGAGATATTGTATTC-3' and reverse, 5'-AAAATT TATACCTTTTTA CTACCTTCGTC-3'; P53 (U) (197 bp) forward, 5'-GTATAGTATATA ATGAGATAT TGTATT TGT-3' and reverse, 5'-AAAATTTATACCTTTTTACTA CCTTCATC-3'; EGFR (M) (172 bp) forward, 5'-TAATTT TAGGTTTGAAGGGGTAGC-3' and reverse, 5'-CAACAC AAAACACAAAATAATAAACG; EGFR (U) (169 bp) forward, 5'-TAATTTTAGGTTTGA AGGGGTAGTG-3' and reverse, 5'-CACAAA ACACAAAATAATAAACAC C-3'; CHFR (M) (213 bp) forward, 5'-TTGCGATGTTTG TTGATAGTAGC-3' and reverse, 5'-GTCCGATAAAAC CCTAACCG-3'; CHFR (U) (219 bp) forward, 5'-GATTTG TGATGTTTGTTGATAGTAGTG-3' and reverse, 5'-AAC ATCCAATAAAACCCTAACCAC-3'; THBS1 (M) (185 bp) forward, 5'-TTTAGTCGTAGTTTTCGAATTTACG-3' and reverse, 5'-ATA ACTAACCAAAACAACACTCGTC-3'; THBS1 (U) (183 bp) forward, 5'-TTAGTTGTAGTTTTT GAATTTATGG-3' and reverse, 5'-TAACTAACCAAAACA ACACTCATC-3'; C-myc,(M) (151 bp) forward, 5'-TTGAAA TTTTAGGTTTTTGAGATCG-3' and reverse, 5'-CGTAAT AAAAAA ACTCATCCACGTA-3'; C-myc (U) (151 bp) forward, 5'-TGAAATTTTAGGTTTTTGAGATTGG-3' and reverse, 5'-CATAATAAAAAAACTCATCCACATA-3'. Amplified PCR products were evaluated by $2 \%$ agarose gel electrophoresis.

\section{Results}

Quarantine of control groups. The results of the PCR amplification of the gag gene were all negative (Fig. 1). This illustrates the fact that the samples of the control group were uninfected.

PCR amplification of DNMTs and MGMT. The results of the agarose gel electrophoresis were as expected (Fig. 2), and the results of DNA sequencing were identical to those of the reference sequence (National Center for Biotechnology Information, Bethesda, MA, USA). The absorbance (A)260/A280 nm value of purified plasmid DNA was between 1.8 and 2.0, which conformed to the requirement of follow-on experiments.

Establishment of standard curves. The standard and melting curves were established by RT-qPCR amplification of the positive standard with a multiple proportion dilution $\left(1 \times 10^{1}-1 \times 10^{6}\right.$ 
Table I. Intra-assay reproducibility of DNMT and tumor-associated genes.

Intra-assay data

\begin{tabular}{|c|c|c|c|c|c|c|}
\hline Gene & Plasmid concentration (ng) & Repeat 1 & Repeat 2 & Repeat 3 & Mean \pm SD & Variation coefficient $(\%)$ \\
\hline 18S-rRNA & 59.33 & 14.52 & 14.37 & 14.25 & $14.38 \pm 0.11$ & 0.76 \\
\hline DNMT1 & 36.01 & 22.02 & 21.85 & 21.99 & $21.95 \pm 0.074$ & 0.38 \\
\hline DNMT3a & 17.67 & 17.58 & 17.32 & 17.53 & $17.48 \pm 0.11$ & 0.63 \\
\hline DNMT3b & 12.67 & 34.56 & 34.30 & 34.27 & $34.38 \pm 0.13$ & 0.38 \\
\hline MGMT & 28.33 & 20.85 & 21.29 & 20.86 & $21.00 \pm 0.21$ & 0.01 \\
\hline P73 & 19.01 & 15.37 & 15.25 & 15.52 & $15.38 \pm 0.11$ & 0.72 \\
\hline P53 & 13.06 & 21.57 & 21.33 & 21.53 & $21.48 \pm 0.11$ & 0.51 \\
\hline KRAS & 27.33 & 24.04 & 23.87 & 23.98 & $23.96 \pm 0.075$ & 0.31 \\
\hline NRAS & 13.67 & 18.35 & 18.23 & 18.51 & $18.36 \pm 0.10$ & 0.54 \\
\hline GADD45G & 33.01 & 19.47 & 19.84 & 19.94 & $19.75 \pm 0.18$ & 0.91 \\
\hline EGFR & 32.09 & 23.94 & 23.83 & 23.92 & $29.90 \pm 0.18$ & 0.60 \\
\hline CHFR & 14.09 & 21.85 & 21.29 & 21.86 & $21.67 \pm 0.12$ & 0.55 \\
\hline C-myc & 17.81 & 17.08 & 16.96 & 17.19 & $17.08 \pm 0.094$ & 0.55 \\
\hline THBS1 & 18.09 & 25.39 & 25.44 & 25.36 & $25.40 \pm 0.033$ & 0.13 \\
\hline CEBPA & 17.08 & 21.29 & 20.85 & 20.86 & $20.00 \pm 0.21$ & 1.05 \\
\hline
\end{tabular}

SD, standard deviation.
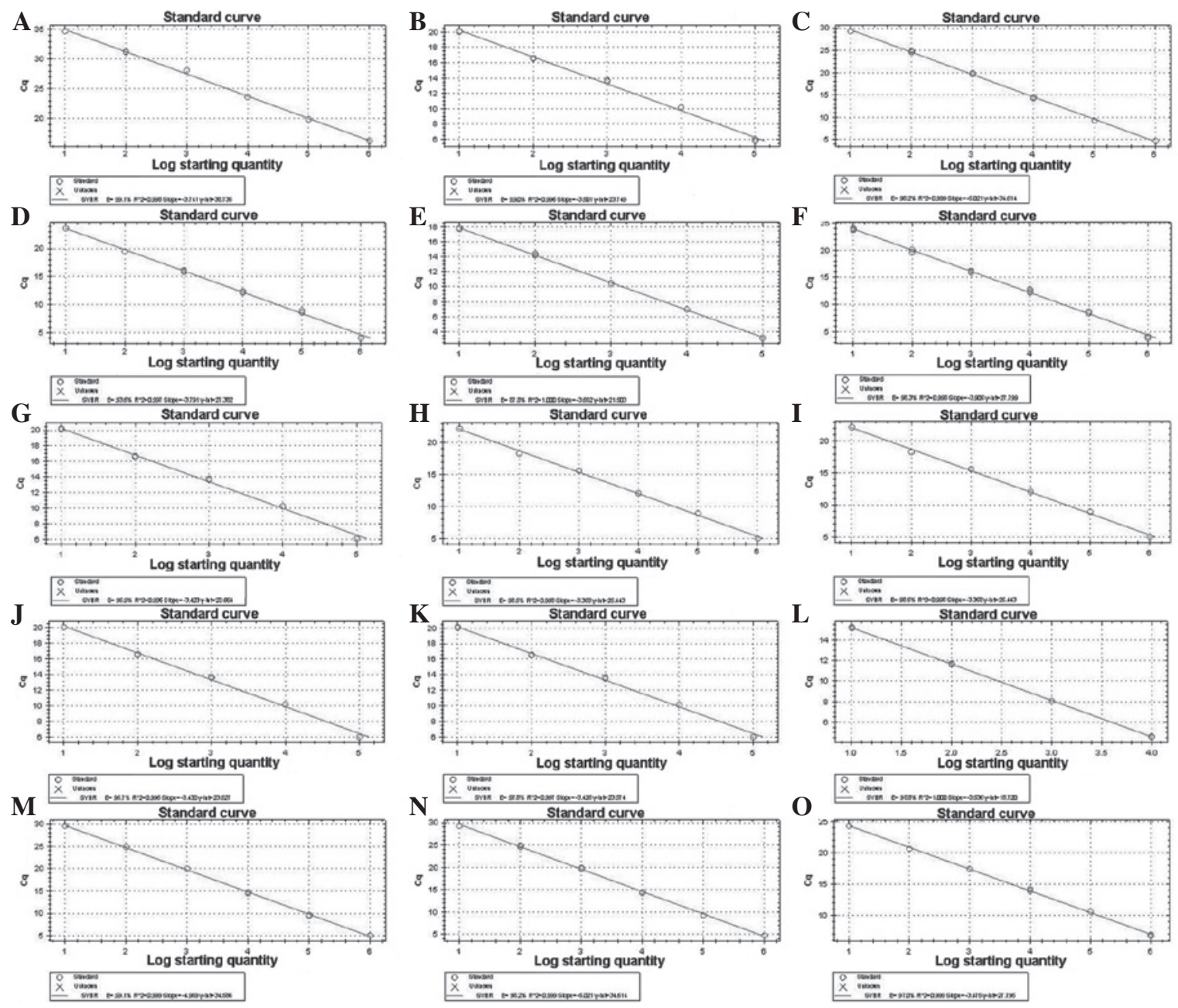

Figure 3. Standard curves of DNMT and tumor-associated genes synthesized by quantitative polymerase chain reaction. (A) 18S-rRNA; (B) DNMT1; (C) DNMT3a; (D) DNMT3b; (E) MGMT; (F) P73; (G) P53; (H) KRAS; (I) NRAS; (J) GADD45G; (K) EGFR; (L) CHFR; (M) C-myc; (N) THBSI; (O) CEBPA. 
Table II. Inter-assay reproducibility of DNMT and tumor-associated genes.

Intra-assay data

\begin{tabular}{|c|c|c|c|c|c|c|}
\hline Gene & Plasmid concentration (ng) & Repeat 1 & Repeat 2 & Repeat 3 & Mean $\pm \mathrm{SD}$ & Variation coefficient (\%) \\
\hline 18S-rRNA & 59.33 & 13.48 & 14.85 & 14.92 & $14.42 \pm 0.19$ & 1.32 \\
\hline DNMT1 & 36.01 & 21.95 & 22.83 & 23.40 & $22.73 \pm 0.59$ & 2.60 \\
\hline DNMT3a & 17.67 & 17.51 & 17.79 & 17.10 & $17.47 \pm 0.28$ & 1.60 \\
\hline DNMT3b & 12.67 & 34.38 & 34.03 & 34.94 & $34.45 \pm 0.64$ & 1.86 \\
\hline MGMT & 28.33 & 20.40 & 21.85 & 21.80 & $21.35 \pm 0.67$ & 3.14 \\
\hline P73 & 19.01 & 21.94 & 20.38 & 21.03 & $21.11 \pm 0.64$ & 3.03 \\
\hline P53 & 13.06 & 22.83 & 21.95 & 23.40 & $22.73 \pm 0.59$ & 2.60 \\
\hline KRAS & 27.33 & 22.83 & 24.38 & 23.90 & $23.70 \pm 0.65$ & 2.74 \\
\hline NRAS & 13.67 & 18.85 & 17.88 & 18.00 & $18.58 \pm 0.41$ & 2.21 \\
\hline GADD45G & 33.01 & 19.92 & 19.48 & 19.85 & $19.75 \pm 0.19$ & 0.96 \\
\hline EGFR & 32.09 & 22.83 & 22.92 & 23.24 & $22.00 \pm 0.17$ & 0.77 \\
\hline CHFR & 14.09 & 23.85 & 22.40 & 23.80 & $23.35 \pm 0.67$ & 2.87 \\
\hline C-myc & 17.81 & 17.10 & 17.51 & 17.79 & $17.46 \pm 0.28$ & 1.60 \\
\hline THBSI & 18.09 & 25.26 & 25.08 & 25.85 & $25.40 \pm 0.33$ & 1.30 \\
\hline CEBPA & 17.08 & 19.41 & 20.79 & 20.85 & $20.35 \pm 0.67$ & 3.29 \\
\hline
\end{tabular}

$\mathrm{SD}$, standard deviation.
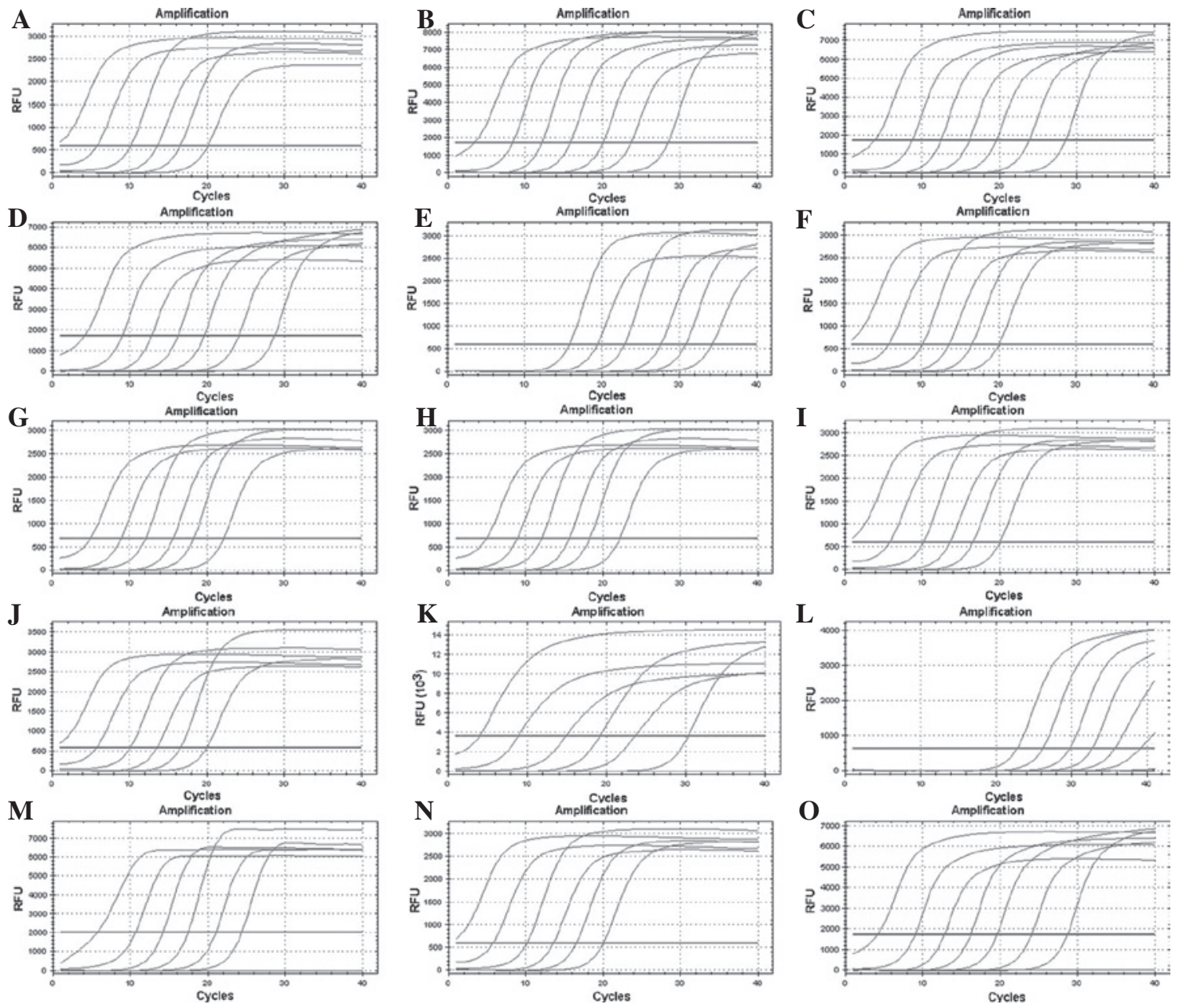

Figure 4. Amplification curves of DNMT and tumor-associated genes synthesized by quantitative polymerase chain reaction. (A) 18S-rRNA; (B) DNMT1; (C) DNMT3a; (D) DNMT3b; (E) MGMT; (F) P73; (G) P53; (H) KRAS; (I) NRAS; (J) GADD45G; (K) EGFR; (L) CHFR; (M) C-myc; (N) THBSI; (O) CEBPA. 
Table III. Relative quantification of DNMT.

Expression of the DNMT mRNA

\begin{tabular}{lccr}
\cline { 2 - 4 } Gene & Cases $(\mathrm{n})$ & $\Delta \Delta \mathrm{Ct}($ mean $\pm \mathrm{SD})$ & \multicolumn{1}{c}{$2^{-\Delta \mathrm{Ct}}$} \\
\hline DNMT1 (control groups) & 20 & $0.00 \pm 0.53$ & $1.00(0.693-1.444)$ \\
DNMT1 (nasal tumor tissues) & 24 & $-0.64 \pm 0.56$ & $1.56(0.678-1.474)$ \\
DNMT3a (control groups) & 20 & $1.01 \pm 0.74$ & $0.497(0.599-1.670)$ \\
DNMT3a (nasal tumor tissues) & 24 & $1.25 \pm 0.73$ & $0.420(0.603-1.659)$ \\
DNMT3b (control groups) & 20 & $1.39 \pm 0.88$ & $0.382(0.503-1.840)$ \\
DNMT3b (nasal tumor tissues) & 24 & $1.25 \pm 0.63$ & $0.420(0.646-1.548)$ \\
MGMT (control groups) & 20 & $0.53 \pm 1.40$ & $0.693(0.379-2.639)$ \\
MGMT (nasal tumor tissues) & 24 & $0.87 \pm 0.75$ & $0.547(0.595-1.682)$
\end{tabular}

SD, standard deviation.
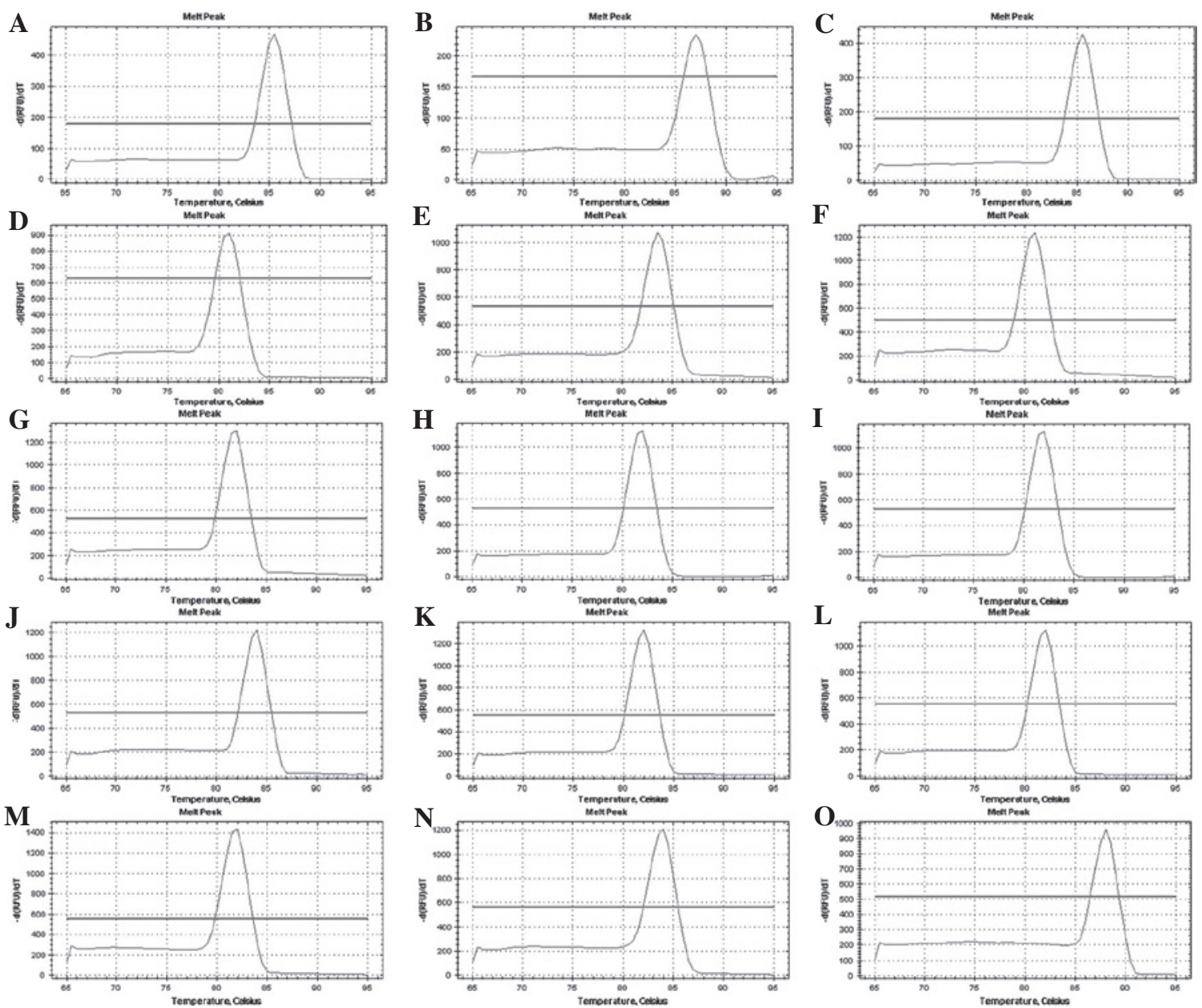

Figure 5. Melting curves of DNMT and tumor-associated genes amplified by quantitative polymerase chain reaction. (A) 18S-rRNA; (B) DNMT1; (C) DNMT3a; (D) DNMT3b; (E) MGMT; (F) P73; (G) P53; (H) KRAS; (I) NRAS; (J) GADD45G; (K) EGFR; (L) CHFR; (M) C-myc; (N) THBSI; (O) CEBPA.

copies $/ \mu \mathrm{l})$. According to the $\mathrm{Ct}$ values of each gene with different dilution degrees, standard curves (Fig. 3) and amplification standard curves (Fig. 4) were produced. As demonstrated in Fig. 4, the correlation coefficient was $>0.999$ and the amplification efficiency was $\sim 1$, which conformed to the requirement of follow-on experiments.
Sensitivity, specificity and repeatability of RT- $q P C R$. Based on the electrophoretic analysis of PCR products (Fig. 2) and the PCR melting curve (Fig. 5), it was concluded that the method had a good specificity as no primer dimers and no additional peaks in the melting curves were observed. The minimum detection concentration was 100 copies $/ \mu 1$ when detected 
Table IV. Relative quantification of goat tumor-associated genes.

Expression of the tumor-associated gene mRNA

\begin{tabular}{|c|c|c|c|}
\hline Gene & Cases (n) & $\Delta \Delta \mathrm{Ct}($ mean $\pm \mathrm{SD})$ & $2^{-\Delta \Delta C t}$ \\
\hline P73 (control groups) & 20 & $0.54 \pm 0.94$ & $0.688(0.521-1.919)$ \\
\hline P73 (nasal tumor tissues) & 24 & $0.65 \pm 0.70$ & $0.637(0.616-1.625)$ \\
\hline P53 (control groups) & 20 & $0.54 \pm 0.35$ & $0.688(0.785-1.275)$ \\
\hline P53 (nasal tumor tissues) & 24 & $1.20 \pm 0.63$ & $0.435(0.646-1.548)$ \\
\hline KRAS (control groups) & 20 & - & - \\
\hline KRAS (nasal tumor tissues) & 24 & - & - \\
\hline NRAS (control groups) & 20 & $-0.69 \pm 0.42$ & $1.613(0.747-1.338)$ \\
\hline NRAS (nasal tumor tissues) & 24 & $-0.68 \pm 0.53$ & $1.602(0.693-1.444)$ \\
\hline GADD45G (control groups) & 20 & $2.48 \pm 0.82$ & $0.179(0.566-1.765)$ \\
\hline GADD45G (nasal tumor tissues) & 24 & $2.47 \pm 0.72$ & $0.180(0.607-1.647)$ \\
\hline EGFR (control groups) & 20 & - & - \\
\hline EGFR (nasal tumor tissues) & 24 & - & - \\
\hline CHFR (control groups) & 20 & $1.02 \pm 0.99$ & $0.493(0.503-1.986)$ \\
\hline CHFR (nasal tumor tissues) & 24 & $1.07 \pm 1.02$ & $0.476(0.493-2.028)$ \\
\hline C-myc (control groups) & 20 & $-0.42 \pm 1.90$ & $1.338(0.268-3.732)$ \\
\hline C-myc (nasal tumor tissues) & 24 & $-2.37 \pm 0.58$ & $5.169(0.669-1.495)$ \\
\hline CEBPA (control groups) & 20 & $-0.85 \pm 0.24$ & $0.555(0.847-1.818)$ \\
\hline CEBPA (nasal tumor tissues) & 24 & $-0.76 \pm 0.26$ & $1.693(0.835-1.197)$ \\
\hline THBS1 (control groups) & 20 & $0.38 \pm 0.25$ & $0.768(0.801-1.189)$ \\
\hline THBS1 (nasal tumor tissues) & 24 & $1.05 \pm 0.32$ & $0.483(0.801-1.248)$ \\
\hline
\end{tabular}

$\mathrm{SD}$, standard deviation.
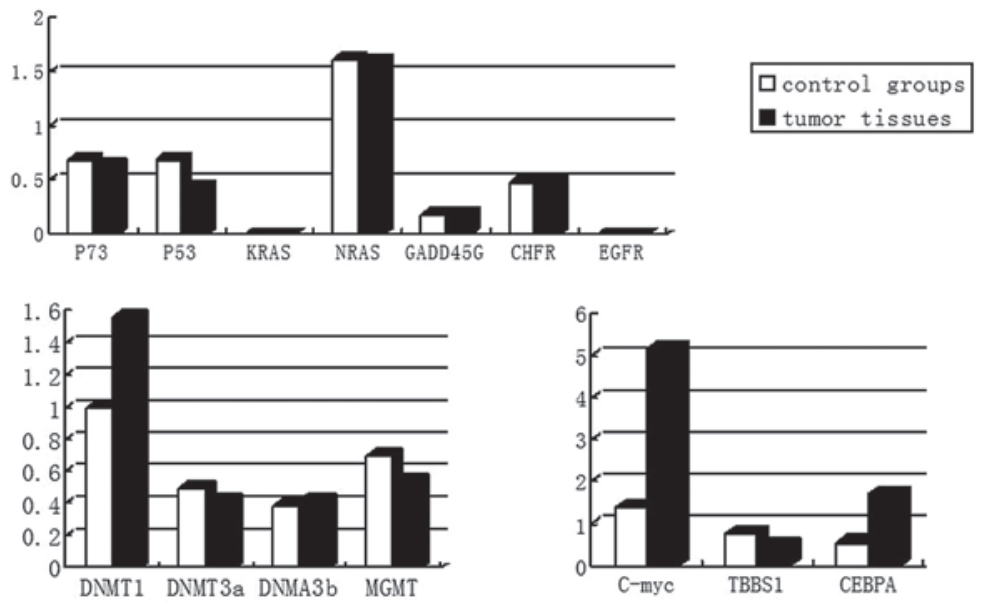

Figure 6. RNA expression of DNMTs and tumor-associated genes in goats. DNMT expression in normal nasal epithelial tissues were used as the control.

with a standard sample of a 10X gradient dilution (Fig. 3). The intra-assay variation coefficient ranged between 0.13 and $1.05 \%$ and the inter-assay variation coefficient was between 0.67 and $3.29 \%$, which suggested that the experiments had improved repeatability (Tables I and II).

mRNA expression of DNMT. The expression levels of DNMT1 were observed to increase by $56 \%$ compared with those in normal nasal epithelial tissues, while MGMT, DNMT3a and
DNMT3b had similar expression levels in the two tissue types (Table III and Fig. 6).

mRNA expression of tumor-associated genes. The expression levels of P53 were reduced by $36.8 \%$ and those of THBS1 by $43 \%$, C-myc expression increased 2.9 -fold and CEBPA increased by 2 -fold compared with that in normal nasal epithelial tissues. GADD45, P7, CHFR and NRAS had similar expression levels in the two tissues. No EGFR or KRAS 
Table V. Methylation status of the tumor-associated genes.

Methylation status of the tumor-associated genes (\%)

\begin{tabular}{|c|c|c|c|c|}
\hline Gene & Cases (n) & Exhaustive methylation & Partial methylation & Unmethylated \\
\hline P73 (control groups) & 20 & $0(0 / 20)$ & $0(0 / 20)$ & $100(20 / 20)$ \\
\hline P73 (nasal tumor tissues) & 24 & $0(0 / 24)$ & $0(0 / 24)$ & $100(24 / 24)$ \\
\hline P53 (control groups) & 20 & $0(0 / 20)$ & $0(0 / 20)$ & $100(20 / 20)$ \\
\hline P53 (nasal tumor tissues) & 24 & $0(0 / 24)$ & $0(0 / 24)$ & $100(24 / 24)$ \\
\hline KRAS (control groups) & 20 & $0(0 / 20)$ & $0(0 / 20)$ & $100(20 / 20)$ \\
\hline KRAS (nasal tumor tissues) & 24 & $0(0 / 24)$ & $0(0 / 24)$ & $100(24 / 24)$ \\
\hline NRAS (control groups) & 20 & $0(0 / 20)$ & $0(0 / 20)$ & $100(20 / 20)$ \\
\hline NRAS (nasal tumor tissues) & 24 & $0(0 / 24)$ & $0(0 / 24)$ & $100(24 / 24)$ \\
\hline GADD45G (control groups) & 20 & $15(3 / 20)$ & $0(0 / 20)$ & $85(17 / 20)$ \\
\hline GADD45G (nasal tumor tissues) & 24 & $62.5(15 / 24)$ & $37.5(9 / 24)$ & $0(0 / 24)$ \\
\hline EGFR (control groups) & 20 & $75(15 / 20)$ & $0(0 / 20)$ & $25(5 / 20)$ \\
\hline EGFR (nasal tumor tissues) & 24 & $87.5(21 / 24)$ & $0(0 / 24)$ & $12.5(3 / 24)$ \\
\hline CHFR (control groups) & 20 & $60(12 / 20)$ & $0(0 / 20)$ & $40(8 / 20)$ \\
\hline CHFR (nasal tumor tissues) & 24 & $58.3(14 / 24)$ & $0(0 / 24)$ & $41.7(10 / 24)$ \\
\hline C-myc (control groups) & 20 & $15(3 / 20)$ & $0(0 / 20)$ & $85(17 / 20)$ \\
\hline C-myc (nasal tumor tissues) & 24 & $87.5(21 / 24)$ & $0(0 / 24)$ & $12.5(3 / 24)$ \\
\hline CEBPA (control groups) & 20 & $40(8 / 20)$ & $0(0 / 20)$ & $60(12 / 20)$ \\
\hline CEBPA (nasal tumor tissues) & 24 & $100(24 / 24)$ & $0(0 / 24)$ & $0(0 / 24)$ \\
\hline THBS1 (control groups) & 20 & $20(4 / 20)$ & $0(0 / 20)$ & $80(16 / 20)$ \\
\hline THBS1 (nasal tumor tissues) & 24 & $100(24 / 24)$ & $0(0 / 24)$ & $0(0 / 24)$ \\
\hline
\end{tabular}

expression was observed in either of the two tissue types (Table IV and Fig. 6).

Promoter methylation status. The amplified products of the positive control were consistent with the anticipated results. The only tumor suppressor genes observed to be methylated were CHFR, GADD45G and THBS1. The methylation expression rate of the CHFR gene was $\sim 60 \%$ in the two tissues. The methylation rate of THBS1 was $100 \%$ in nasal tumor tissues and $20 \%$ in normal nasal epithelial tissues. The exhaustive methylation expression rate of GADD45G was $62.5 \%$ and the partial methylation expression rate was $37.5 \%$ in nasal tumor tissue, while no methylation was observed in the normal nasal epithelial tissues. C-myc was the only proto-oncogene observed to be methylated. The methylation expression rate of C-myc was 87.5 and $15 \%$ in the nasal tumor tissues and normal nasal epithelial tissues, respectively. The methylation expression rate of CEBPA was $100 \%$ in the nasal tumor tissues and $40 \%$ in normal nasal epithelial tissues. EGFR had similar expression levels in the two tissues. The methylation expression rate of the EGFR gene was $\sim 80 \%$ in the two tissues (Table V, Figs. 7 and 8).

\section{Discussion}

The present study established a method to screen animals for ENT using RT-qPCR analysis. In the PCR analyses performed, pure single products were obtained and agarose gels appeared according to the expected outcome. No primer dimers and no unexpected peaks in the melting curves were observed, and DNA sequencing results were similar to those of the reference sequence, thus suggesting that the experiments were specific, repeatable and accurate. The correlation coefficients of each gene were $>0.999$, which indicated the $\mathrm{Ct}$ value and concentration of cDNA had a strong linear association $(8,9)$. The amplification efficiency was $\sim 1$, which indicated that RT-qPCR products were of high quality. In terms of repeatability of the experiments, the intra-assay and inter-assay variation coefficient remained $<3.29 \%$, which demonstrated that the method was repeatable and may be used to detect gene transcription (8).

DNMT1, DNMT3a and DNMT3b are the encoding genes of methyltransferase $(3,10)$. DNA methylation involves the addition of a methyl group to the carbon in 5-position of the cytosine pyrimidine ring via the action of DNMT1, DNMT3a and DNMT3b $(3,4,10)$. The clustering of the $\mathrm{CpG}$ island of upstream genes often leads to the downregulation of the expression of the downstream gene. Abnormal methylation has been previously suggested to be one of the mechanisms of tumorigenesis (11). A previous study demonstrated that the DNMT1 gene was closely associated with DNA methylation and that DNMT1 is important in the maintenance of methylation (12). The MGMT gene is a tumor suppressor gene, and a previous study identified that abnormal expression of the MGMT gene resulted in the activation of the oncogene or inactivation of tumor suppressor genes, thus leading to tumor formation (13). Previous studies have identified an association between the MGMT gene and occurrence, development, 


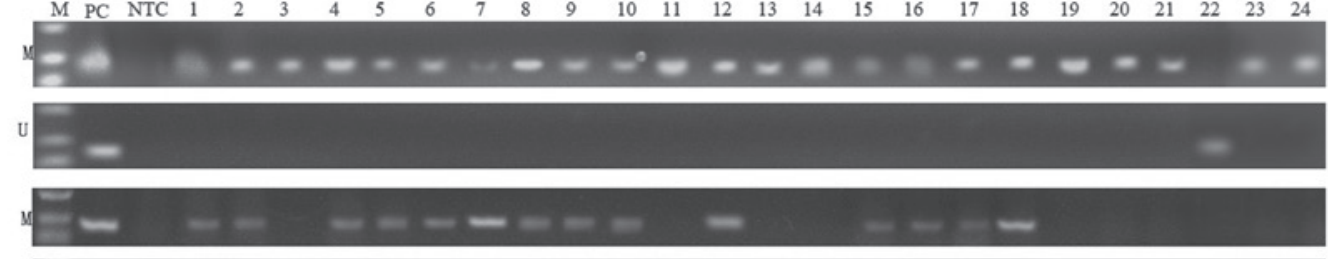

B

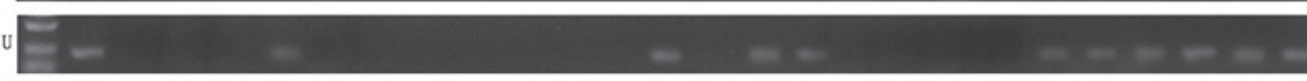

C

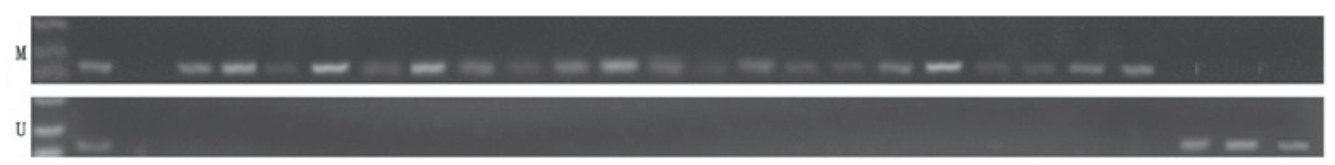

D

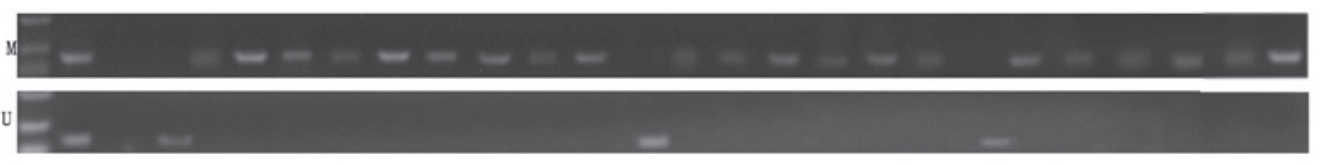

$\mathbf{E}$
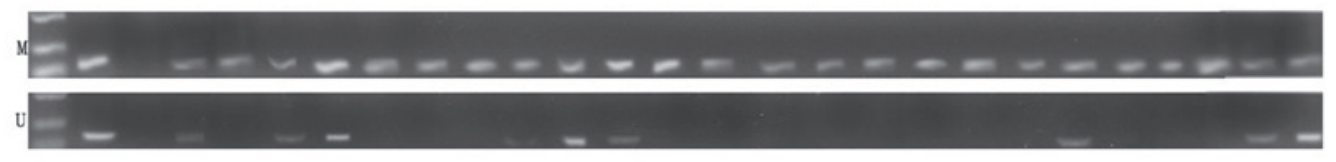

F

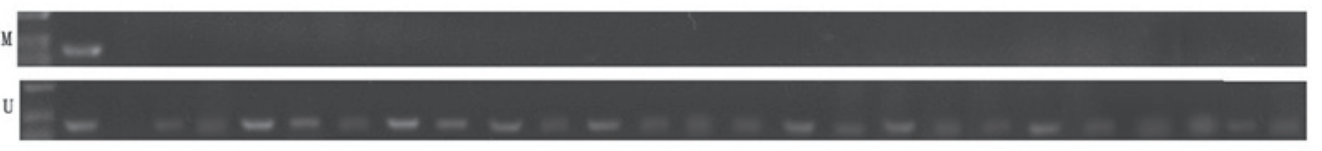

G

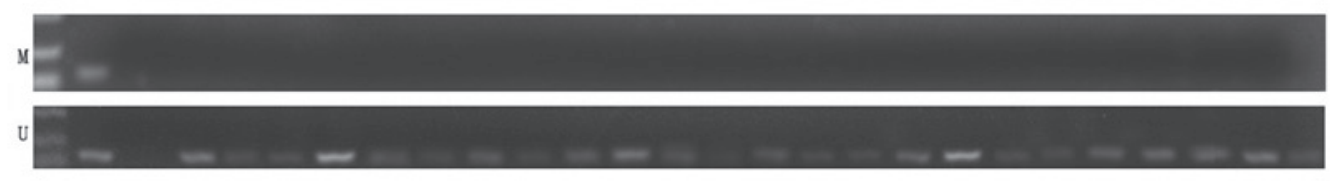

H

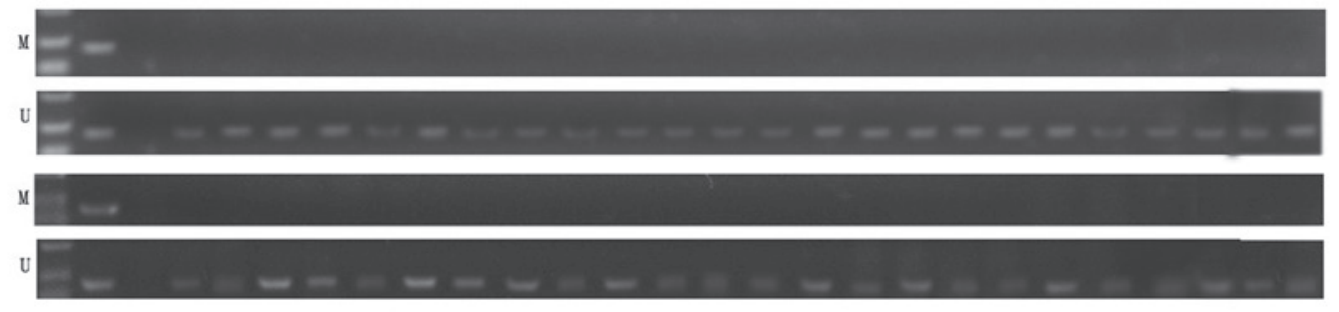

$\mathbf{J}$

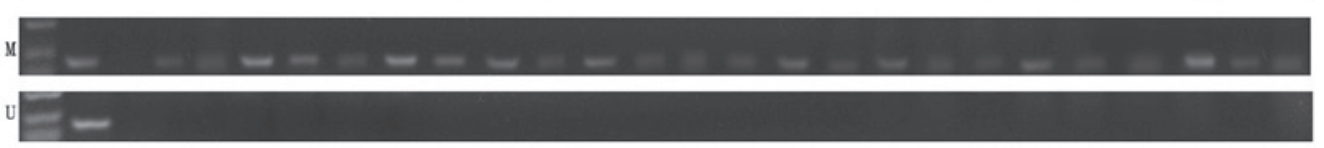

Figure 7. Methylation expression rates of tumor-associated genes in nasal tumor tissues. (A) CEBPA; (B) CHFR; (C) C-myc; (D) EGFR; (E) GADD45G; (F) KRAS; (G) NRAS; (H) P53; (I) P73; (J) THBS1. M, methylation; U, unmethylated; PC, positive control; NTC, negative control; 1-24, 24 nasal tumor tissue samples.

biological behavior and prognosis of malignant tumors (13-15). In the present study, MGMT, DNMT3a and DNMT3b were observed to have similar expression levels in the two tissues, while the expression of DNMT1 was upregulated in nasal tumor tissues. A total of six out of ten tumor-associated genes were observed to exhibit methylation in the present study. The presence of methylation was suggested to be associated with abnormal expression levels of DNMT1 (12).

A previous study demonstrated that the inactivation of tumor suppressor genes is important in the development of nasopharyngeal carcinoma (16). The inactivation of anti-oncogenes by methylation is more common in nasopharyngeal carcinoma (16). Nasopharyngeal cancer-associated gene methylation involves the entire process of cell cycle regulation, DNA repair, cell apoptosis and tumor metastasis (16). It has been demonstrated that DNA methylation is one of three mechanisms of tumor suppressor gene inactivation, while in certain cases, DNA methylation is the only mechanism of tumor suppressor gene inactivation (17). P53 has been reported to be absent or mutated in $\sim 50 \%$ of human tumors (18). Once induced, P53 protein can combine with regulatory sequences and a series of trans-activating genes (p2 J, GADD45) and act as a transcriptional regulatory factor $(18,19)$. Gopisetty et al $(20)$ observed that abnormal methylation of P53 and p14ARF may lead to activation and expression of various apoptosis-promoting genes and induce cell apoptosis. The hypermethylation of tumor suppressor genes, tumor metastasis suppressor genes, hormone receptor genes, DNA repair genes and angiogenesis-inhibiting gene promoters can result in the downregulation of expression or loss of the corresponding gene (19). THBS1 was first observed in platelet particles and can be expressed by tumor cells, macrophages, mononuclear cells and endothelial cells (19). THBS1 
A

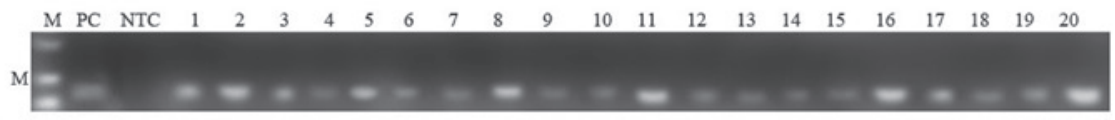

A

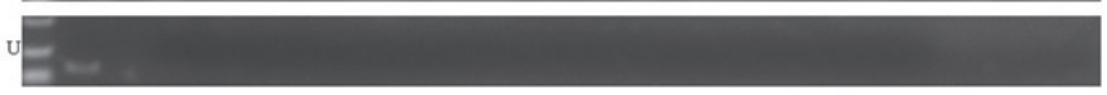

B

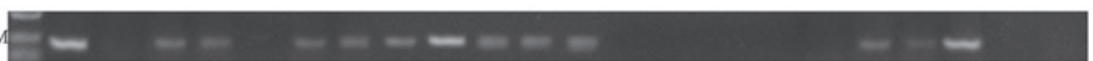

B

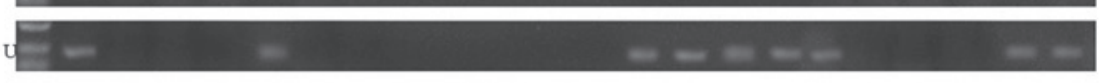

C
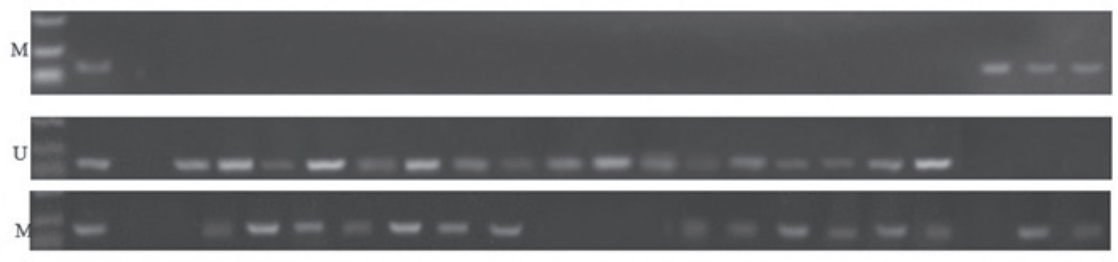

D

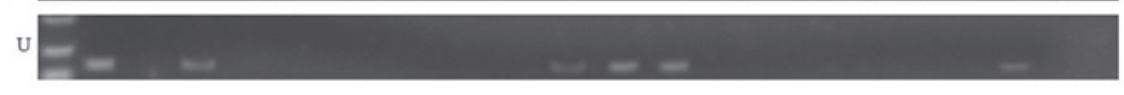

$\mathbf{E}$

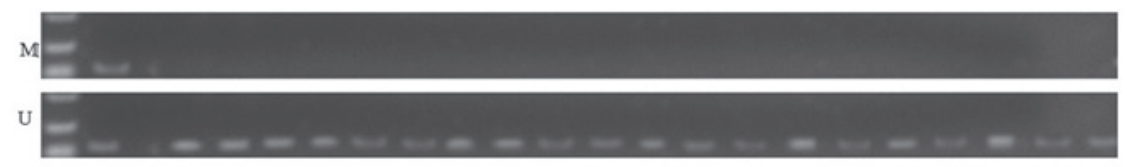

F
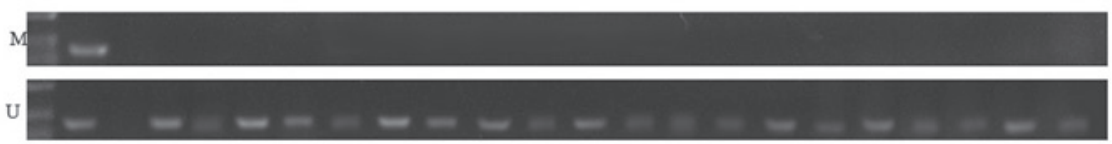

G

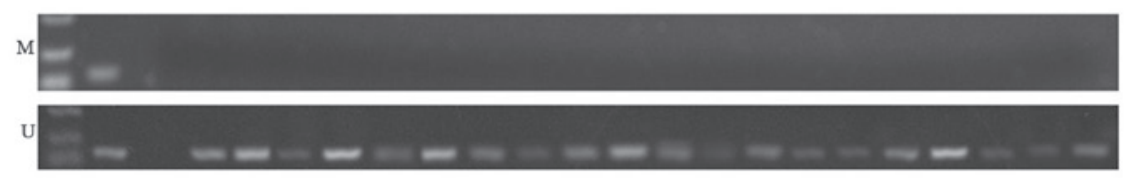

H
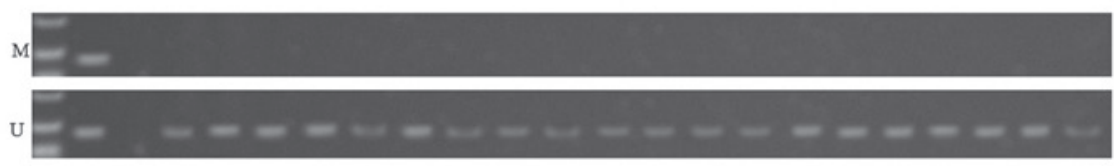

I

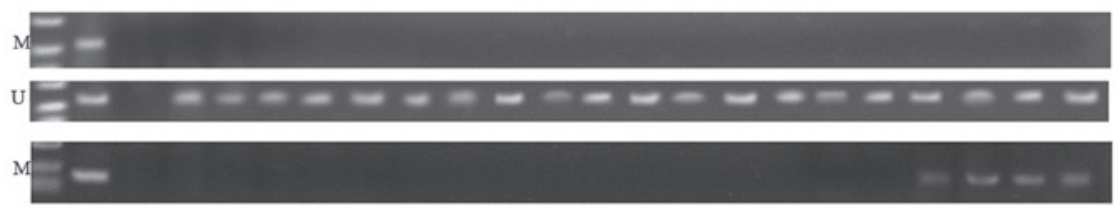

$\mathbf{J}$

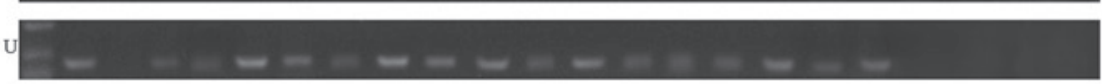

Figure 8. Methylation expression rates of tumor-associated genes in normal nasal epithelial tissues. (A) CEBPA; (B) CHFR; (C) C-myc; (D) EGFR; (E) GADD45G; (F) KRAS; (G) NRAS; (H) P53; (I) P73; (J) THBS1. M, methylation; U, unmethylated; PC, positive control; NTC, negative control; 1-24, 24 nasal tumor tissue samples.

is one of the strongest negative regulators of angiogenesis (21), which can additionally regulate cell adhesion, migration, proliferation and differentiation and induce platelet aggregation (22). Previous studies have demonstrated that THBS1 participates in the development and prognosis of neoplasms and the expression levels are negatively correlated with tumor progression (23). In the present study, methylation was identified to be absent in the P73 and P53 genes. The methylation expression rate of THBS1 was $100 \%$ in nasal tumor tissues and $20 \%$ in normal nasal epithelial tissues. The exhaustive methylation expression rate of GADD45G was $62.5 \%$ and the partial methylation expression rate was $37.5 \%$ in nasal tumor tissue, while no methylation was observed in normal nasal epithelial tissues. The expression levels of P53 were reduced by $36.8 \%$ and those of THBS1 were reduced by $43 \%$.
Proto-oncogene activation is an important mechanism in the development of various malignant tumor types (24). Point mutations (25) and low methylation levels, for example, can lead to abnormal activation of proto-oncogenes, which increases the proliferative and survival abilities, thus promoting tumor progression (26). C-myc is a multi-functional cancer gene, and as it has transcription factor activity, it can activate cell proliferation, inhibit cell differentiation, regulate the cell cycle and regulate cell apoptosis (26). Abnormal methylation can result in abnormally high expression levels of C-myc and thus lead to the occurrence and development of tumors (26). In the present study, the methylation expression rate of C-myc was $87.5 \%$ in nasal tumor tissues and $15 \%$ in normal nasal epithelial tissues. The expression levels of C-myc increased 2.9-fold compared with those in normal nasal epithelial tissues. 
The CEBPA gene and transcription factor C/EBP $\alpha$ exhibit important effects in the process of regulation of myeloid differentiation and proliferation (27). Abnormal expression levels of the CEBPA gene at a transcriptional and translational level have been reported to influence the occurrence of cancer (27). In the present study, the methylation expression rate of CEBPA was observed to be $100 \%$ in nasal tumor tissues and $40 \%$ in normal nasal epithelial tissues. The expression levels of CEBPA increased 2-fold compared with normal nasal epithelial tissues.

EGFR is composed of an extracellular section, transmembrane region and an intracellular kinase activity area (28). Overexpression of EGFR is associated with tumor cell metastasis and invasion (28). In the present study, the methylation expression rate of EGFR gene was $\sim 80 \%$ in the two tissue types, whereas no expression of EGFR was identified.

In conclusion, the expression of DNMT1, C-myc and CEBPA was upregulated, and the expression of P53 and THBSI was downregulated in nasal tumor tissues. Abnormal methylation of the C-myc, CEBPA, GADD45G and THBS1 genes was observed in nasal tumor tissues. The occurrence of ENT may be associated with the abnormal expression and methylation of DNMT1, C-myc, CEBPA, P53, GADD45G and THBS1. Therefore, it is suggested that these six genes may be used as diagnostic marker candidate genes for ENT. The results of the present study can provide a foundation for screening of tumor-specific markers for early diagnosis of ENT and further research into the epigenetic mechanisms of ENTV-induced nasal epithelial cell carcinoma.

\section{References}

1. Kawasako K, Okamoto M, Kurosawa T, et al: Enzootic intranasal tumour virus infection in apparently healthy sheep in Japan. Vet Rec 157: 118, 2005.

2. Pan SY: DNA methylation as a biomarker and its clinical application on molecular diagnosis and treatment. J Mol Diagn Ther 1: 145-147, 2009.

3. He M: DNA methylation and tumor. J Med Mol Biol 8: 641-645, 2011.

4. Kappler JW: The kinetics of DNA methylation in cultures of a mouse adrenal cell line. J Cell Physiol 75: 21-31, 1970.

5. Wassenegger M: RNA-directed DNA methylation. Plant Gene Silencing. Springer 83-100, 2000.

6. Feng YW, Yan QG, Guo WZ, Wang XY and Shu L: Construction and bioinformatics analysis of cDNA library of goat enzootic nasal tumor virus SC strain. Chin Vet Sci 41: 126-130, 2011

7. Wang XY, Feng YW, Yan QG, et al: Cloning and sequence analysis of gag variable region of enzootic nasal tumor virus and endogenous retrovirus of goats. Prog Vet Med 32: 29-32, 2011

8. Zhao YP, Bai DY, LI B, Huang JL, Zhang YH and Mang L: establishment of a real-time RT-PCR assay based on SYBR Green I for detection of the expression of horse toll-like receptor genes. Acta Vet Zootech Sin 44: 220-227, 2013.
9. Xiu JS, Chen XQ, Wang B and Li T: Establishment of a real-time RT-PCR method based on SYBR Green Ifor diagnosis of porcine kobuvirus. Chin J Zoonoses 28: 922-926, 2012.

10. Jeltsch A: Beyond Watson and Crick: DNA methylation and molecular enzymology of DNA methyltransferases. Chembiochem 3: 274-293, 2002.

11. Wang ZK and Wang YF: DNA methvlation and tumor. J Med Postgra 6: 641-645, 2012

12. Liu B, Gu LP, Xing CP, et al: Clinical significance and association of caveolin-1 and Dnmt1 gene with carcinogenesis, development of gastric carcinomas. World Chin J Digestol 17: 1561-1566, 2009.

13. Sun YH, Zhang YZ, Wang ZC, Sun MZ and Zhao DH: Relationship between the expression of O6-methylguanine-DNA methyltransferase in glioma and the survival time of patients. Ai Zheng 23: 1052-1055, 2004 (In Chinese).

14. Esteller M, Toyota M, Sanchez-Cespedes M et al: Inactivation of the DNA repair gene O6-methylguanine-DNA methyhransferase by promoter hypermethylation is associated with $\mathrm{G}$ to $\mathrm{A}$ mutations in K-ras in colorectal tumorigenesis. Cancer Res 60: 2368-2371, 2000

15. Preuss I, Eberhagen I, Haas S, et al: O6-methylguanine-DNA methyhransferase activity in breast and brain tumors. Int J Cancer 61: 321-326, 1995 .

16. Ni HF, Huang GW, Zhang Z, Jiang P and Li Y: Promter methyhlation and the mRNA expression of p73 gene in human nasopharyngeal carcinoma. J Med Res 40: 24-28, 2011.

17. Jones PA and Laird PW: Cancer-epigenetics comes of age. Nat Genet 21: 163-167, 1999.

18. Vogelstein B, Lane D and Levine AJ: Surfing the p53 network. Nature 408: 307-310, 2000.

19. Li M and Wang X: The biological effects of $\mathrm{p} 53$ methylayion profile change. Int Genet ISTIC 35: 243-247, 2012.

20. Gopisetty G, Ramachandran K and Singal R: DNA methylation and apoptosis. Mol Immunol 43: 1729-1740, 2006.

21. Zhang YC, Chen SP, Li J, Wang XD, Deng CS, Zhu YQ and Gong L: $\mathrm{CpG}$ is 1sland methylation and expression of thrombospondin 1gene in colorectal adenocarcinoma. World Chin J Digestol 13: 189-193, 2005.

22. Tsuchida T, Kijima H, Tokunaga T, et al: Expression of the thrombospondin 1 receptor CD36 is correlated with decreased stromal vascularisation in colon cancer. Int J Oncol 14: 47-98, 1999.

23. Maeda K, Nishiguchi Y, Kang SM, et al: Expression of thrombospondin-1 inversely correlated with tumor vascularity and hematogenous metastasis in colon cancer. Oncol Rep 8: 763-766, 2001.

24. Jiang BY: Clinical relevance and underlying mechanism of altered expression of proto-oncogene BCL 11A in non-small cell lung cance. Southern Medical University 2010.

25. Konopka JB, Watanabe SM, Singer JW, et al: Cell lines and clinical isolates derived from $\mathrm{Ph} 1$-positive chronic myelogenous leukemia patients express $\mathrm{c}$-abl proteins with a common structural alteration. Proc Natl Acad Sci USA 82: 1810-1814, 1985.

26. Zhang Y, Li JS, Guo MZ, Meng BS, Chen XY and Zhang JP: Effects of S-ademetionine on proliferation of gastric cancer cell lines and methylation of c-myc and Upa genes. Chin J Dig 30: 322-326, 2010

27. Wang LMM, Xiao HW and Huang H: Roles of CEBPA mutation and expression abnormality in acute myeloid leukemia. Zhongguo Shi Yan Xue Ye Xue Za Zhi 20: 1256-1260, 2012 (Aritcle in Chinese).

28. Wang XF, Du ZW, Wu M, Zhang YC, Jiang Y and Zhang GZ: DNA extraction from formalin-fixed and paraffin-embedded tissues by triton X-100 for effective amplification of EGFR gene by polymerase chain reaction. Chem Res Chin Univ 25: 501-505, 2011. 